\title{
Finding the Perfect Match: Different Heavy-Duty Mobile Applications Call for Different Actuators
}

\author{
David Fassbender *(1) and Tatina Minav (1) \\ Tampere University, Faculty of Engineering and Natural Sciences, IHA-Innovative Hydraulics and Automation \\ * Correspondence: david.fassbender@tuni.fi
}

Received: date; Accepted: date; Published: date

\begin{abstract}
For the longest time, valve-controlled, centralized hydraulic systems have been the state-of-the-art technology to actuate heavy-duty mobile machine (HDMM) implements. Due to the typically low energy efficiency of those systems, a high number of promising, more-efficient actuator concepts has been proposed by academia as well as industry over the last decades as potential replacements for valve control-e.g. independent metering, displacement control, different types of electro-hydraulic actuators (EHAs), electro-mechanic actuators or hydraulic transformers. This paper takes a closer look on specific HDMM applications for these actuator concepts to figure out where which novel concept can be a better alternative to conventional actuator concepts, and where novel concepts might fail to improve. For this purpose, a novel evaluation algorithm for actuator-HDMM matches is developed based on problem aspects that can indicate an unsuitable actuator-HDMM match. To demonstrate the functionality of the match evaluation algorithm, four actuator concepts and four HDMM types are analysed and rated in order to form 16 potential actuator-HDMM matches that can be evaluated by the novel algorithm. The four actuator concepts comprise a conventional valve-controlled concept and three different types of EHAs. The HDMM types are excavator, wheel loader, backhoe and telehandler. Finally, the evaluation of the 16 matches results in 16 mismatch values of which the lowest indicates the "perfect match". Low mismatch values could be found in general for EHAs in combination with most HDMMs but also for a valve-controlled actuator concept in combination with a backhoe. Furthermore, an analysis of the concept limitations with suggestions for improvement is included.
\end{abstract}

Keywords: Hydraulic Actuators; Heavy-Duty Mobile Machines; Electro-Hydraulics; Design Process, Energy Efficiency

\section{Introduction}

The actuation of heavy-duty mobile machines (HDMMs) is a unique field of research. Especially the mainly linear actuators for HDMM implements, which fulfill the actual work task, must generally meet particular requirements, such as high safety, robustness, compactness and low price. So far, valve-controlled centralized hydraulic actuator systems have been the state-of-the-art technology to fulfill these requirements. Different valve-control actuator concepts exist, such as load sensing (LS) systems, negative flow control or open-center systems [1], but they all have the presence of high metering losses in common. The resulting low energy efficiency is problematic, facing rising energy prices but especially the tightening emission legislation in many countries all over the world. As a result, academia as well as industry have proposed numerous novel actuator concepts over the last decades as alternatives to valve-controlled actuators on HDMMs. Main examples are independent metering [2], multi-pressure rail systems [3], displacement control [4], electro-hydraulic actuators (EHAs)[5], hydraulic-transformer-based systems [6], digital hydraulics [7] and even non-hydraulic electro-mechanic actuators [8]. Moreover, just for EHAs themselves, further 8 subclasses could be identified by Ketelsen et al. [5]. 
The result of this development trend can be seen in Figure 1: One the on hand, a high number of actuator concepts exists-different conventional valve-controlled and novel concepts-on the other hand, a variety of different HDMM types has to be considered for the application of these actuator concepts. The class of HDMMs covers machines from small road-sweapers or skidsteers to big combine harvesters or mining excavators. Between those machines, power levels, number of actuators, environments, work cycles and other application factors can vary significantly. Thus, there cannot be an ideal one-fits-all actuator concept for HDMMs, and each potential actuator-HDMM match has to be evaluated separately. As can be seen in Figure 1, this results in a very high number of potential actuator-HDMM matches—each red line represents one-that need to be evaluated to get a complete overview.

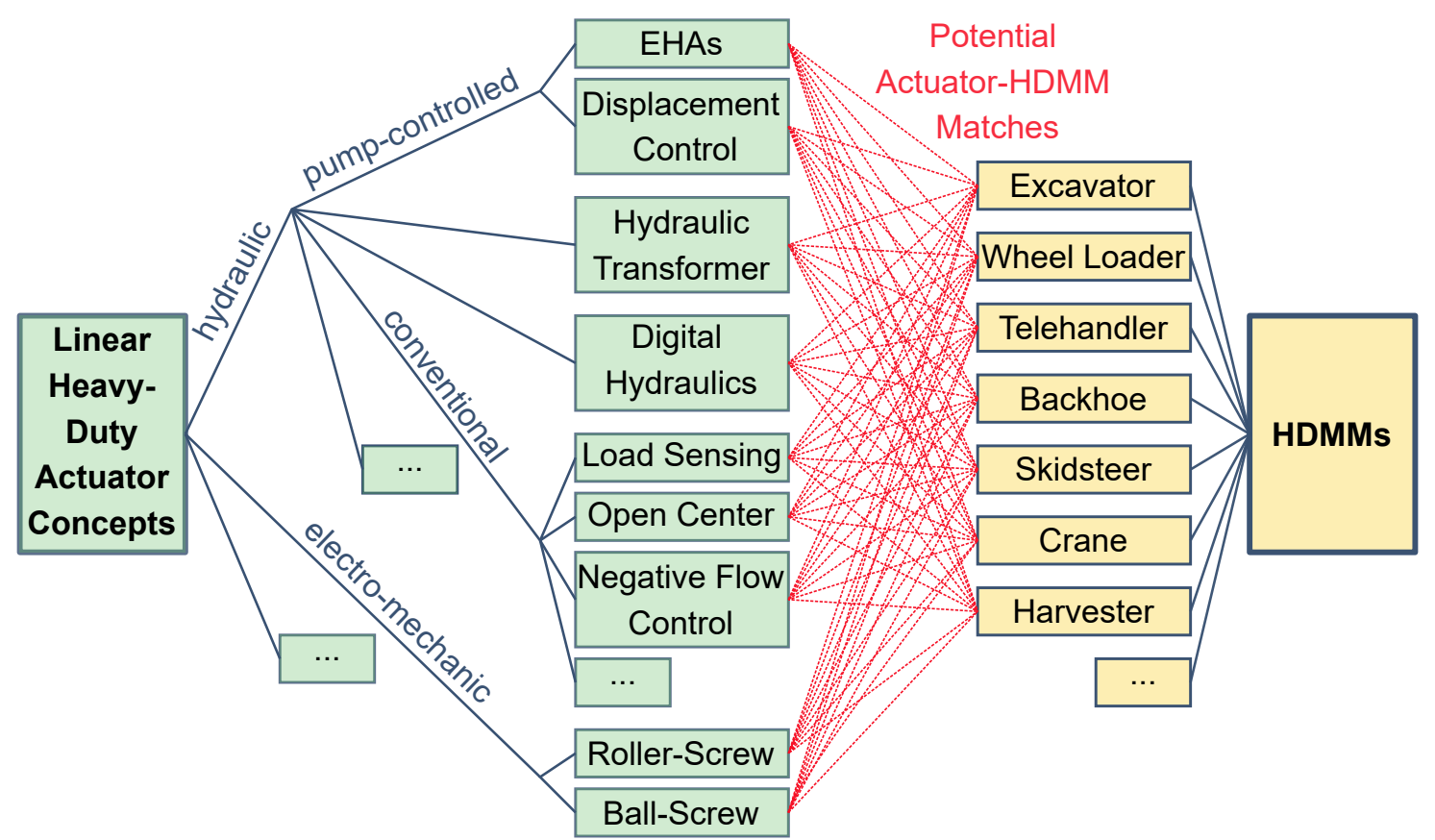

Figure 1. A rough, incomplete classification of linear heavy-duty actuator concepts and of HDMMs as well as the visualization of the resulting potential actuator-HDMM matches

With traditional design-process methods such as decision/evaluation matrices, each match had to be evaluated separately, which would require a lot of effort. Therefore, this paper proposes a novel approach to numerically evaluate multiple actuator-application (in this case HDMM) matches with low effort at the same time. This can help HDMM manufacturers to easily select the best option out of multiple actuator concepts for their next product generation; on the other hand, actuator suppliers can identify suitable applications for their actuator products. Furthermore, the novel evaluation algorithm is modular and allows to add more actuator concepts or HDMM types to the evaluation process later on.

The paper is structured as follows: First, the novel numerical match evaluation concept is proposed alongside the specific application on HDMMs. Second, four different representative actuator concepts are analysed and rated in order to be evaluated by the novel algorithm. Third, the paper rates four HDMM types for the evaluation, and forth, potential matches between the rated HDMMs types and actuator concepts are evaluated with the novel algorithm and discussed. Finally, limitations that apply to the concept are analysed and addressed in the last section before the conclusion. 


\section{Machine-Actuator-Match Evaluation Concept}

\subsection{Definition of a "Good Match"}

To create a novel match evaluation concept, first, the fundamentals that make an actuator-application match a good match have to be known. The general assumption is that the development of every new actuator concepts and respectively achieving a better application-actuator match is based on solving problems that other actuator concepts show. The best application-actuator match is the match with the least problems. An example for a problem aspect is "energy losses", which lead to increased energy costs and emissions. Conventional valve-controlled actuator concepts have the characteristic "causing high energy losses"; so a new actuator concept is developed with the characteristic "causing low energy losses" in order to reduce the problem aspect "energy losses".

However, an issue might be that the new actuator concept has higher costs per actuator than the old concept and thus increases another problem aspect "high capital costs". Consequently, each actuator concepts can improve but also worsen problem aspects and will only be a promising alternative or a better match if over all the problem improvement is more significant than the problem worsening. This highly depends on the application or HDMM, respectively. Improving the problem "energy losses" is more significant for a HDMM with high energy turnover than for a machine with low energy energy consumption, and high costs per actuator are more problematic for a HDMM with a lot of actuators than for a HDMM with only a few.

The best actuator-HDMM match is determined by being least problematic concerning a set of problem aspects. This can be identified by looking at the problematic actuator characteristics concerning each problem aspect and the HDMM attribtes that define how significant each problem aspect is.

\subsection{Evaluation Algorithm}

After developing this general definition, it is step-by-step numerically applied for the match evaluation:

1) For each actuator-HDMM-match evaluation $n \in \mathbb{N}$ problem aspects are taken into account.

2) For each problem aspect $i \in[1 . . n]$, a problematic actuator characteristic and an HDMM attribute exist.

3) Every actuator concept receives a number $c_{i} \in[0 . .5]$ where 0 means the problematic actuator characteristic for problem aspect $i$ is "not applying" and 5 it is "strongly applying".

4) For each HDMM type, the numbers $a_{i} \in[0 . .5]$ mean that the HDMM attribute for problem aspect $i$ is 0 "not expressed" to 5 "stronly expressed".

5) If $c_{i}$ and $a_{i}$ are multiplied, the problem degree of the match concerning problem aspect $i$ is obtained.

6) Summing up the problem degrees for all $n$ aspects, the so-called mismatch value mmv results.

7) Finally, among multiple possible matches, the match with the highest mismatch value is the worst, and the match with the lowest mismatch value is the best or "perfect match".

For convenient handling, the evaluation concept is expressed as a vector operation in Equation 1. The mismatch value is calculated by the scalar product of a HDMM-attribute vector $\boldsymbol{a}$ and an actuator-characteristics vector $c$. The vector $\boldsymbol{a}$ contains HDMM attribute expressions for each of the $n$ problem aspect and the vector $c$ the $n$ problematic actuator characteristics respectively. Due to the nature of the scalar product, the result equals the sum of all $n$ problem degrees.

$$
m m v=\boldsymbol{a} \bullet \boldsymbol{c}=\left(\begin{array}{c}
a_{1} \\
a_{2} \\
\vdots \\
a_{n}
\end{array}\right) \cdot\left(\begin{array}{c}
c_{1} \\
c_{2} \\
\vdots \\
c_{n}
\end{array}\right)=a_{1} \cdot c_{1}+a_{2} \cdot c_{2}+\ldots+a_{n} \cdot c_{n}
$$




\subsubsection{Considered Match Aspects}

The quality of the match evaluation depends highly on choosing the right set of problem aspects. No important problem aspects should be neglected, and at the same time, unessential aspects should be avoided because they would decrease the influence of the other, more significant aspects. Furthermore, the right set of aspects depends on the range of actuator concepts that are supposed to be evaluated. The design engineer has to consider every potential application problem of the actuator concepts that are evaluated and formulate them in the form of problem aspect, problematic actuator characteristic and HDMM attribute. This requires sufficient knowledge of the technologies and should be ideally done by a group of experienced engineers. For this paper, a first approach of identifying the essential aspects for the actuator concepts in Section 3 is presented in Table 1. The problematic nature of those aspects for certain actuator concepts will become more apparent after the analyses in Section 3.

Table 1. Considered problem aspects for the match evaluation

\begin{tabular}{|c|c|c|c|}
\hline$i$ & problem aspect & HDMM attribute $\left(a_{i}\right)$ & $\begin{array}{l}\text { problematic actuator } \\
\text { characteristic }\left(c_{i}\right)\end{array}$ \\
\hline 1 & $\begin{array}{l}\text { high component costs for } \\
\text { small or rarely used } \\
\text { actuators }\end{array}$ & $\begin{array}{l}\text { high number of actuators } \\
\text { with low energy } \\
\text { consumption }\end{array}$ & high price per actuator \\
\hline 2 & $\begin{array}{l}\text { achieving large actuator } \\
\text { sizes }\end{array}$ & containing large actuators & issues with larger actuators \\
\hline 3 & power distribution costs & $\begin{array}{l}\text { long distances between } \\
\text { actuators and main body of } \\
\text { HDMM }\end{array}$ & $\begin{array}{l}\text { high costs per supply-line } \\
\text { length }\end{array}$ \\
\hline 4 & $\begin{array}{l}\text { achieving high actuator } \\
\text { power }\end{array}$ & $\begin{array}{l}\text { high actuator power } \\
\text { requirements }\end{array}$ & power-upscaling issues \\
\hline 5 & energy losses & $\begin{array}{l}\text { high energy turnover of } \\
\text { implements in general and } \\
\text { compared to other machine } \\
\text { functions like driving }\end{array}$ & $\begin{array}{l}\text { low single actuator energy } \\
\text { efficiency }\end{array}$ \\
\hline 6 & $\begin{array}{l}\text { energy losses due to } \\
\text { simultaneous actuation }\end{array}$ & $\begin{array}{l}\text { high amount of } \\
\text { simultaneous actuator } \\
\text { operation }\end{array}$ & $\begin{array}{l}\text { low energy efficiency during } \\
\text { simultaneous operation of } \\
\text { actuators }\end{array}$ \\
\hline 7 & $\begin{array}{l}\text { making use of energy } \\
\text { recuperation }\end{array}$ & high amount of load braking & $\begin{array}{l}\text { no/low recuperation } \\
\text { potential }\end{array}$ \\
\hline 8 & overheating of components & $\begin{array}{l}\text { potentially medium to high } \\
\text { environment temperatures }\end{array}$ & low cooling capability \\
\hline
\end{tabular}

\subsubsection{Not Scalable Factors}

Another aspect that is not covered by the concept so far is that problem aspects such as aspect 1 in Table 1, the actuator costs, might not only scale with the extend of the HDMM attribute expression but also involve a fixed, application-independent term. In the example of aspect 1 , this could be the costs of a centralized supply for a centralized hydraulic actuator concept, which is almost independent from the HDMM attribute, number of actuators. Therefore, a fixed problem term $f_{i} \in[0 . .5]$ can be added for each problem aspect that has an application-independent component. Equation 2 shows this for aspect 1 in Table 1 and is also the final form of the evaluation algorithm used in Section 5.

$$
m m v=a \bullet c+f_{1}
$$

\section{Characterisation of Heavy-Duty Actuator Concepts}

This section analyses a few exemplary actuator concepts and rates their problematic characteristics in the form of a vector $c$ as described in the previous section. 


\subsection{Valve-Controlled Load-Sensing Actuators}

Valve-controlled actuators are the current state-of-the-art concept to drive HDMM implements, and the LS concept is among the most common forms of supplies for those actuators. Therefore, the concept is chosen for this paper to represent a state-of-the-art baseline.
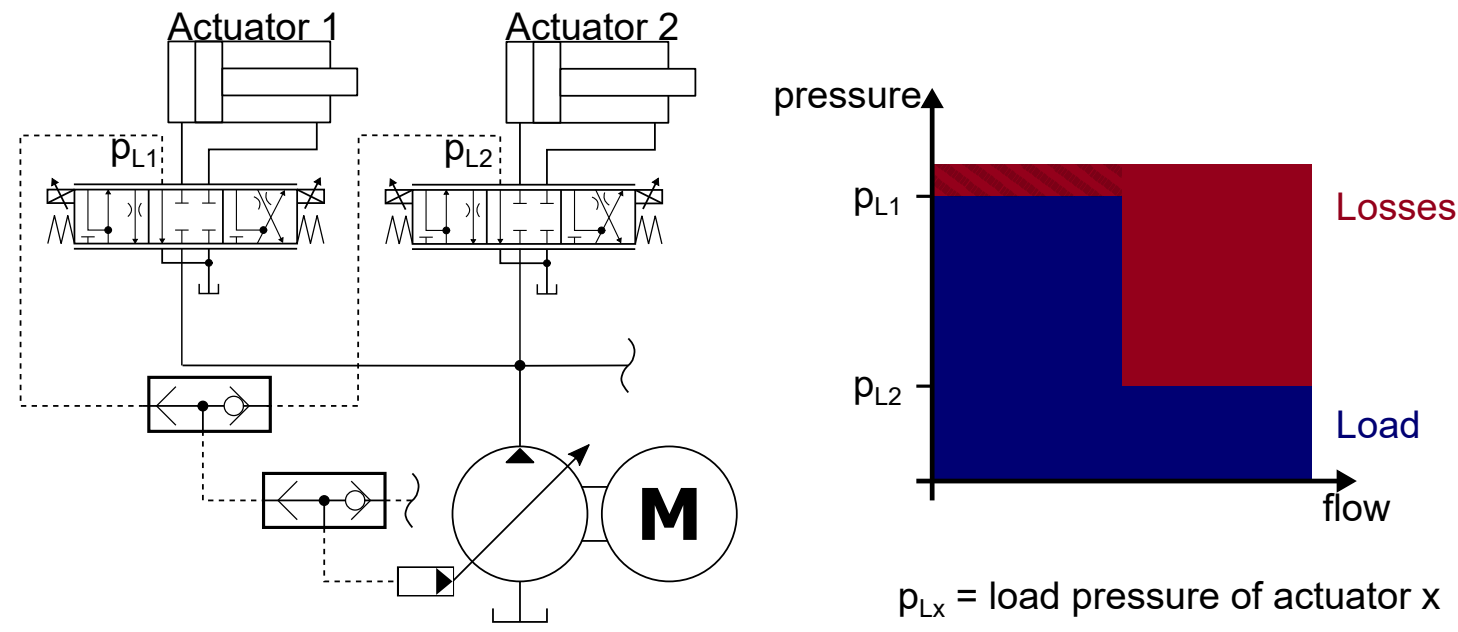

Figure 2. Simplified load sensing system with two actuators (but option for more) and the related pressure-flow diagram for a specific load situation

The simplified schematics of a LS system in Figure 2 is used to analyse the characteristics vector of this concept (Equation 4). The LS supply can be formed by a constant-speed motor (e.g. combustion engine) and variable-displacement pump, or a variable-speed motor (e.g. electric) and a fixed-displacement pump. Furthermore, each actuator requires its own control valve(s). A main advantage is that one LS supply can be used for multiple actuators and only needs to be scaled up for more flow if actuators have to operate simultaneously at high speeds. Moreover, the concept is very mature, and standardized, compact valve assemblies are available at low prices. Thus, adding more actuators requires minimal financial effort and $c_{1}$ is low (1). On the other hand, the fixed costs for the LS supply are rather high, which is expressed by:

$$
f_{1, L S}=4
$$

Because the size of the actuator only influences the required supply flow, bigger actuators can be easily handled by choosing components with higher nominal flow, and $c_{2}$ is rated 1 . Since the system is centralized, expensive hydraulic high-pressure lines need to connect each actuator with the supply, which leads to a high $c_{3}$ (3). For higher power demands, pump and motor can easily be increased in size, and LS systems can often be found on large HDMMs with high power demands. Thus, $c_{4}$ is rated low (1).

In terms of energy efficiency, the pressure-flow diagram in Figure 2 provides more insights. If actuator 1 was operated alone, only the small shaded loss area would be present and the system efficiency relatively high, which is why $c_{5}$ is still rated medium (3). Regardless, the efficiency can be extremely low when another actuator with lower load pressure is operated simultaneously such as actuator 2 in Figure 2. In this case, the load pressure difference is compensated by throttling valves, and the power represented by the red area is dissipated into heat. Accordingly, the aspect 6 and factor $c_{6}$ were introduced especially for valve-controlled actuator concepts that generally all have a highly problematic characteristic concerning this aspect. $c_{6}$ is thus rated 3 (not worse because the effect is only significant if the load pressures differ a lot).

Furthermore, braking energy can only be dissipated by valves in this system and not recuperated, which leads to a high $c_{7}$ value (5). However, cooling is easy because the centralized design allows to 
utilise a common cooler for all actuators together. Thus, $c_{8}$ is rated 2 but not 1 because the cooler still requires extra costs, space and energy.

$$
\boldsymbol{c}_{L S}^{\top}=\left(\begin{array}{llllllll}
1 & 1 & 3 & 1 & 3 & 3 & 5 & 2
\end{array}\right)
$$

\subsection{Electro-Hydraulic Actuators}

In research, EHAs are one of the most focused concepts for replacing conventional actuator concepts on HDMMs and, therefore, considered here as well. The common principle is using electric variable-speed motors in combination with fixed-displacement pumps in direct connection to a single actuator. This allows to control the pump flow and thus the actuator flow and speed. A detailed review of this actuator concept can be found in [5], where 8 subclasses of EHAs are defined. For this paper, three different types of EHAs are considered for the match evaluation, which can be seen in Figure 3 .

a)

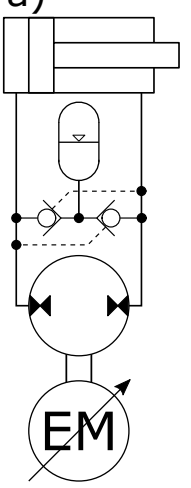

b)

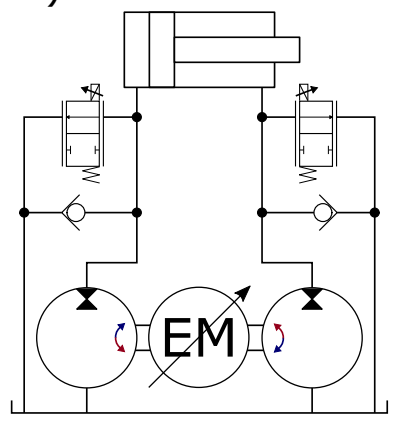

c)

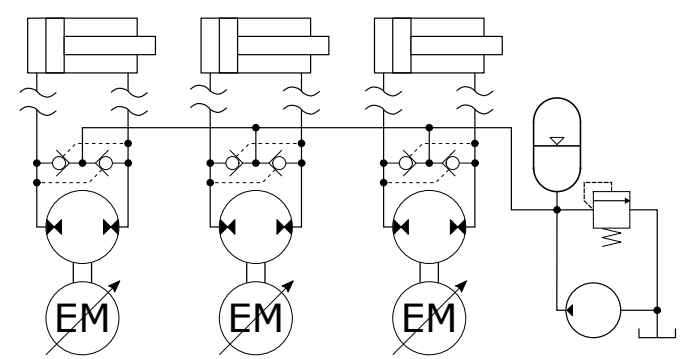

Figure 3. Simplified schematics of considered EHA types a) compact single pump b) compact dual pump according to [9] and c) single-pump centralized

\subsubsection{Baseline Electro-Hydraulic Actuator: Compact Single-Pump Type}

The single-pump EHA is one of the most common EHA concepts and thus chosen as a baseline EHA here. For the other two EHA concepts, only the differences to this baseline EHA are discussed. Figure 3a shows typical schematics of this type. Due to the unbalanced flow between symmetrical pump and asymmetric cylinder, compensating valves are required. The accumulator provides the pendulum volume and pressure to avoid cavitation.

Because each actuator requires its own electric motor, pump and flow compensation mechanism, the costs for additional actuators $c_{1}$ (in Equation 6) are high (4). One the other hand, there are no components that cause fixed costs except for the energy supply which is necessary for each actuator concept anyway and

$$
f_{1, \text { EHASinglePump }}=1 \text {. }
$$

Very large or long actuators are critical because the pendulum volume is large in these cases as well, and the accumulator size must be rapidly increased [10]. Therefore, $c_{2}$ is rated 4 . The only necessary supply line for these actuators is an electric cable. Because long electric cables are significantly less expensive than hydraulic high-pressure lines, $c_{3}$ is only rated 1.

Power can be scaled up by using bigger pumps and motors, which is less problematic. A minor issue can be the lack of flow-compensating valves that can handle higher flows, and two valves in parallel might need to replace a single valve [11]. Thus, $c_{4}$ is rated 2. Efficiencies of these actuators are relatively high - at least compared to valve-controlled actuators. In [12], hydraulic circuit efficiencies of $75 \%$ could be achieved for different EHAs in experiments. Accordingly, $c_{5}$ is rated 2 . Because, the actuators are individual, and each have their own pump, simultaneous actuation has no influence on 
the efficiency and $c_{6}$ is 0 . Energy can be electrically recuperated, which is only compromised by the hydraulic circuit efficiency and the need for additional electric hardware; therefore, $c_{7}$ is rated 2.

In [13], it was shown that passive cooling of these actuators can be sufficient for low powers (max $1.5 \mathrm{~kW}$ in experiments) and at medium ambient temperature. However, for higher power levels common for HDMMs, active cooling is necessary. This requires a cooler for each actuator because the actuators have no hydraulic connection, which is expensive and results in a high $c_{8}(4)$.

$$
\boldsymbol{c}_{\text {EHASinglePump }}^{\top}=\left(\begin{array}{llllllll}
4 & 4 & 1 & 2 & 2 & 0 & 2 & 4
\end{array}\right)
$$

\subsubsection{Compact Dual-Pump Electro-Hydraulic Actuator}

Using two pumps of different size for each actuator allows to compensate the differential cylinder flow with the pumps themselves. The additional valves in Figure $3 \mathrm{~b}$ only manage small flows to avoid cavitation or over-pressurization due to slight mismatches between pump and cylinder ratios [9]. The additional pump makes the EHA more expensive and $c_{1}$ is increased to 5 . On the other hand, no pressurized reservoir is necessary and a vented tank instead of an accumulator can be used. This makes bigger actuators less problematic and reduces $c_{2}$ to 1 . At the same time, it might be necessary to install the tank further away from the cylinder because the tank must remain in a stable orientation in order to avoid leakage. Thus, $c_{3}$ is increased to 2 . Scaling up the power is less problematic because only the pumps, not the valves need to be increased in size $\left(c_{4}=1\right)$. The other characteristics do not vary from the baseline EHA:

$$
\begin{gathered}
\boldsymbol{c}_{\text {EHADualPump }}^{\top}=\left(\begin{array}{llllllll}
5 & 1 & 2 & 1 & 2 & 0 & 2 & 4
\end{array}\right) \\
f_{1, \text { EHADualPump }}=1
\end{gathered}
$$

\subsubsection{Centralized Electro-Hydraulic Actuator}

Centralising multiple EHAs by installing the main circuit components on the rear of the HDMM with a common low-pressure supply and long hydraulic lines supplying the actuators has multiple effects on the characteristics compared to the baseline EHA: First, the common low pressure supply increases the fixed costs, and

$$
f_{1, \text { EHACentralized }}=3,
$$

but the price per actuator decreases, and $c_{1}$ becomes smaller. Furthermore, larger actuators are less problematic because a very large accumulator can still be acceptable when it is mounted on the rear of the vehicle and not next to the cylinder. Thus, $c_{2}$ is reduced to 3 . Moreover, long hydraulic lines across the HDMM are more expensive than electric cables and $c_{3}$ increased to 3 .

The maximum efficiency will decrease because more line losses are present [14] and the charge pump causes losses. This leads to a higher $c_{5}$ (3). Regardless, a further benefit is the cooling capability. Instead of using a separate cooler for each EHA, a single cooler can be located in the common low-pressure supply, which is more convenient and reduces $c_{8}$ to 2 . Other characteristics do not change compared to the baseline EHA:

$$
c_{\text {EHACentralized }}^{\top}=\left(\begin{array}{llllllll}
3 & 3 & 3 & 2 & 3 & 0 & 2 & 2
\end{array}\right)
$$

\section{Classification of Heavy-Duty Mobile Machine Types}

In this section, the attributes of four typical HDMM types are analysed in order to use them for the match evaluation in section 5. This requires the definition of attribute vectors $\boldsymbol{a}$. 


\subsection{Compact Excavators}

Excavators are one of the most common HDMMs and their attributes are representative for a number of similar HDMMs. Moreover, the here considered compact excavator EC15E from Volvo [15] is a typical representative of a low-power HDMM. Shield and bucket are both actuator functions with a low energy turnover because they are rarely actuated or see low loads, while boom and stick actuators have higher energy demands; thus, $a_{1}$ is rated 2. For other excavators with actuators for e.g. boom adjustment or special tools, this number could be rated higher. Due to the compact size of the machine, the actuators are rather small as well, and $a_{2}$ is rated 1 . Furthermore, most actuators are located close to the main vehicle body, but the bucket actuator is mounted at the end of the relatively long excavator arm. Accordingly, $a_{3}$ is chosen to be 2 .

On the exemplary excavator, each actuator can be supplied with up to $10 \mathrm{~kW}$ of hydraulic power [15], which is relatively low; thus, $a_{4}$ is rated 2 . Moreover, typical excavator tasks, such as truck loading, digging or grading, involve mainly the linear implement actuators and less driving. As a result, the attribute $a_{5}$, representing the relative energy consumption of the implements, is rated high (5). Furthermore, analyses of work cycles show that simultaneous actuation of multiple work functions is common especially for expert operators; thus, $a_{6}$ is rated 5 .

The amount of load braking, which can be used for recuperation, always depends on the work task. Grading does not offer a lot of recuperation potential, but most other tasks are cyclic and involve up and down movement. Down movement can be used for recuperation, but typically the boom is lifted with load, the load is dumped and then the boom is lowered empty, which offers less potential energy. Still, the potential $a_{7}$ is rated high 3 , but could be adjusted if a specific work cycle is supposed to be considered for the match evaluation. The attribute $a_{8}$ can also only be specified if a concrete application is known and is thus rated with a neutral/medium value of 3 here. The resulting attribute vector is

$$
\boldsymbol{a}_{\text {compactExcavator }}=\left(\begin{array}{llllllll}
2 & 1 & 2 & 2 & 5 & 5 & 4 & 3
\end{array}\right) .
$$

\subsection{Mid-Size Wheel Loader}

The actuated implement parts of wheel loaders, such as the here considered Volvo LH90 [16], typically comprise boom, shovel, steering and sometimes an additional tool. All of those are frequently actuated and see medium to high loads during common work cycles. Accordingly, $a_{1}$ is rated low (2). Especially the tilt cylinders can be large in diameter (cylinder bore of $180 \mathrm{~mm}$ at a stroke of $430 \mathrm{~mm}$ [16]); thus, $a_{2}$ is rated 3 . Because the boom is rather short, all actuators are relatively close to each other and the main vehicle body, which leads to a low $a_{3}$ (2). On the considered conventional wheel loader [16], a single actuator can be supplied with up to $88 \mathrm{~kW}$ of hydraulic power, which is considered high and leads to a high $a_{4}(4)$. Because common wheel loader work cycles like truck loading or Y-cycles involve significant amounts of driving, $a_{5}$ is only rated medium (3). Simultaneous operation of boom and shovel cylinders is common but not necessarily constantly the case, which leads to $a_{6}$ being 3 . Lowering the boom involves braking against gravity, which can be used for recuperation, but the boom is most often lowered empty, and lowering makes up only a short part of common work cycles, which involve more driving instead. Accordingly, $a_{7}$ is rated 3. $a_{8}$ is rated neutral (3) because the specific application environment is not known. The resulting attribute vector is

$$
\boldsymbol{a}_{\text {wheelLoader }}=\left(\begin{array}{llllllll}
2 & 3 & 2 & 4 & 3 & 3 & 3 & 3
\end{array}\right) .
$$

\subsection{Backhoe Loader}

Backhoes, such as the Case 580 Super N [17] considered here, combine a lot of different implement functions. The loader function and excavator function cannot be used at the same time and are therefore frequently in standby. Furthermore, many auxiliary functions require cylinder actuators but 
are only used once in a while: side supports, boom swing, excavator telescope arm, bucket gripper and others. All in all, this means a high number of actuators with low energy turnover and a high $a_{1}(5)$.

Because backhoes are rather compact, actuator sizes are medium or small (more than half of the cylinders have bore diameters smaller than $100 \mathrm{~mm}$ [17]) and $a_{2}$ is rated 2. Furthermore, actuators are located close to the main body $\left(a_{3}=2\right)$. Moreover, up to $65 \mathrm{~kW}$ of hydraulic power is available but can most likely not be used for the small actuators entirely, and $a_{4}$ is rated 3. No specific work cycle is considered here because backhoes are too versatile; however, driving is one of the main functions. Accordingly, $a_{5}$ is rated medium (3). Actuation of more than one actuator at the same time is also common for backhoes, and $a_{6}$ is rated 3 .

The main implement parts, excavator boom and loader boom, move vertically; thus, there is a potential for energy recuperation, but the masses are commonly rather low. Therefore, $a_{7}$ is rated 3. $a_{8}$ is rated neutral (3) because the specific application environment is not known. The resulting attribute vector is

$$
\boldsymbol{a}_{\text {backhoe }}=\left(\begin{array}{llllllll}
5 & 2 & 2 & 3 & 3 & 3 & 3 & 3
\end{array}\right) .
$$

\subsection{Telehandler}

Telehandlers represent another type of HDMMs that is characterised by long-stroke actuators. The standard implement actuators of telehandlers such as the MHT 10130 [18], which is considered for this analysis, are for steering, boom actuation, tool tilting and extension. They are typically all frequently actuated, and $a_{1}$ is rated 1 . For other telhandler versions with actuators for auxiliary functions like side supports, slope-compensation or grippers, the value would be higher.

Characteristic is the telescopic actuator, which is large in volume due to the long stroke of more than $7 \mathrm{~m}$ [18]. Accordingly, $a_{2}$ is rated 5. Furthermore, the tilt actuator, which extends as well, requires a long and flexible connection to the main part of the vehicle, which results in a high $a_{3}$ (5). Moreover, the actuators can be supplied with $90 \mathrm{~kW}$ of hydraulic power, which is significant and leads to a high $a_{4}$ (4). Similarly to wheel loaders, telehandler work cycles typically involve a significant amount of driving, and $a_{5}$ is rated medium (3). Simultaneous operation of multiple actuators is common for telehandlers as well but not necessarily constantly the case $\left(a_{6}=3\right)$.

The load braking and thus recuperation potential of telehandlers can be considered high because they are often equipped with forks to handle pallets or other compact objects. These objects typically cannot be dumped, and the telehandler must lower the heavy objects again. Compared to the empty lowering typical for HDMMs with bucket or shovel, the potential energy and recuperation potential are much higher in this case, and $a_{7}$ is rated $4 . a_{8}$ is rated neutral (3) because the specific application environment is not known. The resulting attribute vector is

$$
\boldsymbol{a}_{\text {telehandler }}=\left(\begin{array}{llllllll}
2 & 5 & 5 & 4 & 3 & 3 & 4 & 3
\end{array}\right) .
$$

\section{Evaluation of Exemplary Matches and Discussion}

For this section, 16 matches have been formed out of the four actuator types analysed in section 3 and the four HDMM types investigated in section 4. Each actuator-HDMM match has been evaluated with the novel algorithm shown in Equation 2, and the resulting mismatch values can be seen in Table 2 . The table is analysed in order to get insights about the match qualities and to make general statements about HDMM-actuator matches.

Marked in green, the best matches for each HDMM type can be seen in Table 2. Except for the backhoe, all HDMMs match best with the dual-pump EHA. This is in line with the idea that this concept is especially suitable for HDMMs because of its high energy-efficiency and suitability for big actuator sizes. At the same time it has to be considered that the other EHA concepts achieve similarly low mismatch values. If the mismatch-value differences are too small, such as for the excavator and 
Table 2. Mismatch values for all 16 actuator-HDMM matches

\begin{tabular}{l|l|l|l|l} 
& $\begin{array}{l}\text { LS valve } \\
\text { controlled }\end{array}$ & $\begin{array}{l}\text { EHA single } \\
\text { pump }\end{array}$ & EHA dual pump & EHA centralized \\
\hline $\begin{array}{l}\text { compact } \\
\text { excavator }\end{array}$ & 71 & 49 & 48 & 51 \\
\hline wheel loader & 58 & 55 & 46 & 53 \\
\hline backhoe & 59 & 61 & 59 & 57 \\
\hline telehandler & 74 & 68 & 56 & 70
\end{tabular}

backhoe, it cannot be said which concept is indeed more suitable for the HDMM because the evaluation algorithm is (at this stage) not trustful enough.

Still, some more tendencies can be identified and statements made where the mismatch values differ: The centralized EHA achieves the lowest mismatch value for the backhoe, which can be explained by the fact that long hydraulic lines are less problematic due to the compact machine design and the high number of actuators that result in high costs for the other EHAs. On the other hand, the algorithm rates the centralized EHA as less suitable for the telehandler, which can be justified, among others, by the high costs for hydraulic high-pressure lines across the large vehicle.

Also significant is the fact that the valve-controlled concept is evaluated to be a far worse match compared to EHAs for all HDMMs except for the backhoe. The low energy efficiency has most likely the biggest influence on that. Still, it is important to notice that the valve-controlled concept seems to be able to keep up with the EHAs on machines like a backhoe. This means for certain HDMMs, introducing novel, supposed-to-be-better actuator concepts might not bring significant benefits after all.

\section{Limitations of Evaluation Concept}

When looking at the evaluation results in the previous section or using the novel algorithm to evaluate other matches, it has to be considered that certain assumptions have been made in order to apply this algorithm, and several limitations exist. Here it should be shortly explained what they are, how they influence the evaluation results and how they could be addressed in the future.

A first, important assumption for the concept is, that only one actuator concept can be used for each HDMM. This is reasonable because using the same type of actuator has positive effects on e.g. costs or maintenance effort, but using two actuator concepts on the same HDMM is still possible. A HDMM could have EHAs for its main actuators and centralized valve-controlled actuators for auxiliary functions. Still, this aspect could be addressed by considering additional "actuator concepts" that are actually combinations out of two other original concepts.

Moreover, it is assumed that all problem aspects can in principle be handled by all actuator concepts with sufficient effort. In fact, for some actuator-HDMM pairings, problems can exist that make a match impossible, such as using electro-mechanical actuators for a HDMM that requires actuators with significantly high, continuous power output [19].

Another issue is that all problem aspects are considered equally in Equation 2. Adding weight factors that give certain problem aspects more influence than others can lead to more valuable results. By adapting the weights the evaluation could also be focused on different objectives such as achieving an especially cheap or especially low-emission match.

An easy step to improve the quality of the results is to include more problem aspects. Not involved yet are the problems of limited space for self-contained actuator concepts, increased weight of actuators, high noise emission or performance issues of various nature.

Finally, it should be noticed that the problematic actuator characteristics and HDMM attributes have been rated by a single engineer in this study with only a few data points and personal knowledge. This might not be objective enough, and if possible, multiple engineers should do the rating and validate their results. Using simulation tools for that process is recommended as well. More advanced 
and objective machine learning methods can unfortunately not be applied. Sufficient data points in form of e.g. data-sheets would exists, but the training required labeled data, which would mean validated mismatch values for match examples. Those could only be obtained with satisfactory trust by doing excessive simulations or experiments of a high number of random actuator-HDMM matches.

\section{Conclusion}

This paper proposes a novel algorithm for the numerical evaluation of matches between linear actuator concepts and HDMM implement applications. The algorithm allows to evaluate fast and with low effort how well a certain actuator concept matches a high number of different HDMM applications, or how well a number of different actuator concepts fit to a certain HDMM, respectively. Furthermore, the evaluation concept is modular and additional actuator concepts or HDMM types can easily be added to the evaluation process. In a next step, 16 matches between four actuator concepts and four HDMM types have been evaluated. The results show that for most machines the three EHA concepts can perform similarly well, while the valve-controlled LS concept can only achieve a good match in combination with a backhoe. Still, this shows, that novel actuator concepts do not necessarily improve the over-all performance of every HDMM, and sticking to conventional concepts can be justified.

Moreover, remaining limitations of the concept were analysed, which demonstrated that current results can only be used for qualitative statements. In order to make quantitative, precise evaluation statements, the concerns of Section 6 should be addressed in a next step. In a second step, more actuator concepts such as displacement-control, electro-mechanical actuators or hydraulic transformers could be included in the evaluation, as well as more HDMM types.

Author Contributions: Conceptualization, D.F.; methodology, D.F.; formal analysis, D.F.; investigation, D.F.; writing-original draft preparation, D.F.; writing-review and editing, D.F. and T.M.; visualization, D.F.; supervision, T.M.; All authors have read and agreed to the published version of the manuscript.

Funding: This project has received funding from the European Union's Horizon 2020 research and innovation programme under the Marie Skłodowska-Curie grant agreement No 858101

Conflicts of Interest: The authors declare no conflict of interest.

\section{Abbreviations}

The following abbreviations are used in this manuscript:

EHA electro-hydraulic actuator

HDMM heavy-duty mobile machine

LS load sensing

\section{References}

1. Padovani, D.; Rundo, M.; Altare, G. The Working Hydraulics of Valve-Controlled Mobile Machines: Classification and Review. Journal of Dynamic Systems, Measurement, and Control 2020, 142. Publisher: American Society of Mechanical Engineers Digital Collection, doi:10.1115/1.4046334.

2. Weber, J. Independent metering systems. International Journal of Hydromechatronics 2018, 1, 91-106.

3. Siefert, J.; Li, P.Y. Optimal Control and Energy-Saving Anaysis of Common Pressure Rail Architectures: HHEA \& STEAM. BATH/ASME 2020 Symposium on Fluid Power and Motion Control; , 2020.

4. Rahmfeld, R.; Ivantysynova, M.; Weber, J. Displacement Controlled Wheel Loader - a simple and clever Solution. Proceedings of the 4th, International fluid power conference; , 2004; pp. 183-196.

5. Ketelsen, S.; Padovani, D.; Andersen, T.; Ebbesen, M.; Schmidt, L. Classification and Review of Pump-Controlled Differential Cylinder Drives. Energies 2019, 12. doi:10.3390/en12071293.

6. Vael, G.; Achten, P.; Potma, J. Cylinder Control with the Floating Cup Hydraulic Transformer. Eighth Scandinavian Conference on Fluid Power; , 2003; pp. 175-190.

7. Linjama, M. Digital Fluid Power - State of the Art. 12th Scandinavian International Conference on Fluid Power; , 2011. 
8. Thomson-Industries. I 20161202TJ, 2016.
Linear Actuators for On/Off-Highway Vehicles CPEN-0005-01A (https://www.thomsonlinear.com/downloads/capabilities

9. Pedersen, H.C.; Schmidt, L.; Andersen, T.O.; Brask, M.H. Investigation of New Servo Drive Concept Utilizing Two Fixed Displacement Units. 9th JFPS International Symposium on Fluid Power in JFPS International Journal of Fluid Power System; , 2014; Vol. 8. doi:10.5739/jfpsij.8.1.

10. Ketelsen, S.; Kolks, G.; Andersen, T.O.; Schmidt, L.; Weber, J. Bootstrap reservoir concepts for electro-hydraulic compact cylinder drives. 12th International Fluid Power Conference; , 2020; Vol. 3, pp. 201-215. doi:https://doi.org/10.25368/2020.109.

11. Schmidt, L.; Ketelsen, S.; Brask, M.H.; Mortensen, K.A. A Class of Energy Efficient Self-Contained Electro-Hydraulic Drives with Self-Locking Capability. Energies 2019, 12. doi:10.3390/en12101866.

12. Michel, S.; Weber, J. Energy-efficient electrohydraulic compact drives for low power applications. Proceedings of Fluid Power and Motion Control 2012; University of Bath: Bath, England, 2012; pp. 94-108.

13. Michel, S.; Weber, J. Prediction of the thermo-energetic behaviour of an electrohydraulic compact drive. 10th International Fluid Power Conference; , 2016; pp. 219-233.

14. Casoli, P.; Scolari, F.; Minav, T.; Rundo, M. Comparative Energy Analysis of a Load Sensing System and a Zonal Hydraulics for a 9-Tonne Excavator. Actuators 2020, 9. Number: 2 Publisher: Multidisciplinary Digital Publishing Institute, doi:10.3390/act9020039.

15. Volvo-Construction-Equipment. EC15E Compact Excavator Data Sheet. (https://www.volvoce.com/-/media/volvoce/global/products/excavators/compact-excavators /brochures/brochure_ec15e_t4f_en_21_20056711_b.pdf?v=DDZCPw accessed 29.10.2020).

16. Volvo-Construction-Equipment. Wheel Loaders L60H, L70H,L90H. (https://www.volvoce.com/-/media/volvoce/global/products/wheel-loaders/wheel-loaders /brochures/brochure_160h_170h_190h_t4f_en_22_voe2240009459.pdf?v=ngRPPw accessed 28.10.2020).

17. Case-Construction. 580SN Backhoe Loader Data Sheet. (https://assets.cnhindustrial.com/casece/nafta /assets/Brochures/Products/Backhoe-Loaders/580-Super-N/BL-580SN-Specs-201810.pdf accessed 29.10.2020).

18. Manitou-BF-SA. MHT 10130 - Telehandler Data Sheet. (https://www.manitou.com/en /p/VOrvHiwAAC4AxE85 accessed 28.10.2020).

19. Hagen, D.; Pawlus, W.; Ebbesen, M.K.; Andersen, T.O. Feasibility Study of Electromechanical Cylinder Drivetrain for Offshore Mechatronic Systems. Modeling, Identification and Control: A Norwegian Research Bulletin 2017, 38, 59-77. doi:10.4173/mic.2017.2.2.

(C) 2020 by the authors. Licensee MDPI, Basel, Switzerland. This article is an open access article distributed under the terms and conditions of the Creative Commons Attribution (CC BY) license (http://creativecommons.org/licenses/by/4.0/). 